\section{P177 WORK PARTICIPATION IN PATIENTS WITH SYSTEMATIC LUPUS ERYTHEMATOSUS: A SCOPING REVIEW}

${ }^{1}$ Birgit Blomjous, ${ }^{2}$ Gayle Gajadin, 'Irene Bultink, 'Alexandre Voskuyl, ${ }^{3}$ Louise Falzon, ${ }^{4}$ Jan Hoving, ${ }^{1,}{ }^{2}$ Marieke ter Wee. ${ }^{1}$ Dept. of Rheumatology, Amsterdam Rheumatology and immunology Center, Amsterdam Infection and Immunity, Amsterdam UMC, Vrije Universiteit Amsterdam, Amsterdam; ${ }^{2}$ Dept. of Epidemiology and Biostatistics, Amsterdam Public Health, Amsterdam UMC, Vrije Universiteit Amsterdam, Amsterdam, The Netherlands; ${ }^{3}$ Center for Personalized Health, Feinstein Institutes for Medical Research, Northwell Health, New York, USA; ${ }^{4}$ Coronel Institute of Occupational Health, Amsterdam Public Health Research Institute, Amsterdam UMC, University of Amsterdam, Amsterdam, The Netherlands

\subsection{6/lupus-2020-eurolupus.219}

Background The treatment of Systemic Lupus Erythematosus (SLE) has improved over the last decades. However, patients are more vulnerable for sick leave, work disability and unemployment compared to the general population. It is therefore important to understand the magnitude of the problem and the factors that might affect work participation in patients with SLE, to evaluate which actions can be taken to limit the burden on a patient and society level. The aim of this research is to summarize the existing literature on 1) the prevalence of work disability, 2) prognostic factors for work participation and 3 ) the effectiveness of interventions to improve work participation in patients with SLE.

Methods A scoping review, using the PRISMA-ScR statement will be conducted. Full-text original articles published before April 2019 were identified by literature search performed in MEDLINE, Cochrane, Embase and CINAHL. Articles in all languages, concerning work participation in patients with SLE, aged $\geq 18$ years were included.

Results In total, 2050 non-duplicate citations were screened. After title/abstract screening, 75 articles were retrieved. Relevant articles will be identified and reference lists will be searched for additional articles by two independent researchers. Information on author(s), year of publication, study location, intervention type, comparator, duration of the intervention, study population, aims of the study, methodology, outcome measures and important results will be collected. The results will be presented at the congress.

Conclusion Based on this scoping review, implications for future research including a research agenda and daily practice will be identified.

\section{P178 LUPUS NEPHRITIS WAS ASSOCIATED WITH LOW BONE MINERAL DENSITY IRRESPECTIVE OF DURATION ON STEROID TREATMENT IN A LARGE UK STUDY OF JUVENILE SYSTEMIC LUPUS ERYTHEMATOSUS}

${ }^{1}$ Anastasia-Vasiliki Madenidou, 1,2 Yasmin Mahfouz, 1,2 Oliver Chang, 1,2 Farah El-Sharnouby, ${ }^{1}$ Charlene Foley, ${ }^{1}$ Coziana Ciurtin. ${ }^{1}$ Centre for Adolescent Rheumatology, University College London, London; ' University College London Medical School, London, UK

\subsection{6/lupus-2020-eurolupus.220}

Background Estimated 10-20\% of all patients with systemic lupus erythematosus (SLE) develop clinical disease before the age of 18 years and are therefore classified as juvenile-onset SLE (JSLE). JSLE patients have a higher prevalence of lupus nephritis (LN) compared to adult-onset $\mathrm{SLE}^{1}$ and decreased long-term survival compared to JSLE patients without $\mathrm{LN}^{2}$ We aimed to identify clinical and laboratory predictors of $\mathrm{LN}$ in JSLE patients by comparing the baseline characteristics of JSLE patients with and without LN.

Methods This is a single-center retrospective study, who included JSLE patients reviewed in our young adult and adolescent clinics after transitioning from paediatric services. All data have been analyzed descriptively. Mann- Whitney U or Chi- square test were performed to compare the characteristics between the patients with and without LN. In addition, we performed uni and multivariate analysis to investigate potential predictor biomarkers for LN.

Results We identified 134 JSLE patients, out of which 45 (34\%) had LN. The baseline characteristics are detailed in table 1. As expected, the highest dsDNA levels ever were found in patients with $\mathrm{LN}(\mathrm{p}=0.022)$. Patients with $\mathrm{LN}$ also had longer disease duration $(p=0.012)$. The overall clinical manifestations and complications did not differ between JSLE patients with or without $\mathrm{LN}$, except for the prevalence of low

Abstract P178 Table 1 Baseline characteristics

\begin{tabular}{llll}
\hline & Lupus nephritis & Without Lupus nephritis & P value \\
\hline Number of patients, n (\%) & $45(36 \%)$ & $89(66.4 \%)$ & \\
Female & $39(86.7 \%)$ & $73(82 \%)$ & 0.624 \\
Age, years & $22(21-26)$ & $21(19-25)$ & 0.092 \\
Age at diagnosis, years & $13(10-15)$ & $13(11.25-15)$ & 0.471 \\
Age at menarche, years & $12.75(11.25-15.75)$ & $12.25(11-14.38)$ & 0.543 \\
Disease duration, years & $10(8-14)$ & $8(6-13)$ & $0.012^{*}$ \\
BMI, kg/m2 & $24.31(20.41-26.13)$ & $23.96(21.69-28.5)$ & 0.726 \\
ANA & $38(84.4 \%)$ & $85(95.5 \%)$ & 0.064 \\
ENA & $24(53.3 \%)$ & $51(57.3 \%)$ & 0.114 \\
Anti- Ro & $15(33.3 \%)$ & $31(34.8 \%)$ & 0.134 \\
Anti- La & $6(13.3 \%)$ & $14(15.7 \%)$ & 0.130 \\
RF & $3(6.7 \%)$ & $18(20.2 \%)$ & 0.114 \\
Highest dsDNA levels ever & $197(32-434)$ & $48(10-294)$ & $0.022^{*}$ \\
\hline
\end{tabular}

Numbers are medians (interquartile ranges) unless otherwise stated. * $p<0.05$ is significant

Abstract P178 Table 2 Clinical manifestations and complications

\begin{tabular}{llll}
\hline & Lupus nephritis & $\begin{array}{l}\text { Without Lupus } \\
\text { nephritis }\end{array}$ & P value \\
\hline Number of patients, $\mathrm{n}(\%)$ & $45(36 \%)$ & $89(66.4 \%)$ & \\
SLICC at last clinical assessment & 0 & $0(0-0.5)$ & 0.872 \\
SLEDAI at last clinical assessment & $2(0-4)$ & $0(0-2)$ & 0.123 \\
BILAG at last clinical assessment & $1(0-1.75)$ & $1(0-1)$ & 0.822 \\
Constitutional symptoms & $26(80 \%)$ & $67(75.3 \%)$ & 0.666 \\
Mouth ulcers & $31(68.8 \%)$ & $61(68.5 \%)$ & 0.258 \\
Skin involvement & $40(88.9 \%)$ & $73(82 \%)$ & 0.150 \\
Arthritis & $37(82.2 \%)$ & $71(79.8 \%)$ & 0.309 \\
Myositis & $5(11.1 \%)$ & $16(18 \%)$ & 0.451 \\
Neuropsychiatric involvement & $15(33.3 \%)$ & $19(21.3 \%)$ & 0.146 \\
Cardiorespiratory involvement & $12(26.7 \%)$ & $20(22.5 \%)$ & 0.669 \\
Gastrointestinal involvement & $8(17.8 \%)$ & $18(20.2 \%)$ & 0.724 \\
Ophthalmic involvement & $4(8.9 \%)$ & $9(10.1 \%)$ & 0.108 \\
Haematological involvement & $26(57.8 \%)$ & $44(49.4 \%)$ & 0.464 \\
Hypertension & $8(17.8 \%)$ & $6(6.7 \%)$ & 0.071 \\
Stroke & $1(2.2 \%)$ & $2(2.2 \%)$ & 1.000 \\
Low bone mineral density & $16(35.5 \%)$ & $11(12.4 \%)$ & $0.008 *$ \\
Malignancy & $1(2.2 \%)$ & $1(1.1 \%)$ & 1.000 \\
\hline Numbers are medians (interquartile ranges) & unless otherwise stated. ${ }^{*} \mathrm{p}<0.05$ is significant
\end{tabular}

